\title{
Maximum efficiency formulation for inductive power transfer with multiple receivers
}

\author{
Quang-Thang Duonga) and Minoru Okada \\ Graduate School of Information Science, Nara Institute of Science and Technology, \\ 8916-5 Takayama-cho, Ikoma, Nara 630-0192, Japan \\ a)thang@is.naist.jp
}

Abstract: This paper presents closed-form expressions of optimal loads that achieve the maximum efficiency for inductive power transfer (IPT) system with multiple receivers. We model the system as a passive $N$-port network and jointly optimize both reactive and resistive components of the loads. The derived expression allows one to predict RF-to-RF efficiency of IPT system with any number of receivers, under any coupling condition and through any type of media.

Keywords: inductive power transfer, multiple receiver, maximum efficiency, optimal load

Classification: Circuits and modules for electronic instrumentation

\section{References}

[1] T. Imura and Y. Hori: "Maximizing air gap and efficiency of magnetic resonant coupling for wireless power transfer using equivalent circuit and Neumann formula," IEEE Trans. Ind. Electron. 58 (2011) 4746 (DOI: 10.1109/TIE.2011. 2112317).

[2] M. Zargham and P. G. Gulak: "Maximum achievable efficiency in near-field coupled power-transfer systems," IEEE Trans. Biomed. Circuits Syst. 6 (2012) 228 (DOI: 10.1109/TBCAS.2011.2174794).

[3] T. Ohira: "Extended k-Q product formulas for capacitive- and inductivecoupling wireless power transfer schemes," IEICE Electron. Express 11 (2014) 20140147 (DOI: 10.1587/elex.11.20140147).

[4] T. Ohira: "Maximum available efficiency formulation based on a black-box model of linear two-port power transfer systems," IEICE Electron. Express 11 (2014) 20140448 (DOI: 10.1587/elex.11.20140448).

[5] T. Zhang, et al.: "Optimal load analysis for a two-receiver wireless power transfer system,” 2014 IEEE Wireless Power Transfer Conf. (2014) 84 (DOI: 10.1109/WPT.2014.6839600).

[6] T. Imura: "Simultaneous wireless power transfer via magnetic resonant coupling to multiple receiving coils," IEEJ Transaction on Industry Applications 134 (2014) 625 (in Japanese) (DOI: 10.1541/ieejias.134.625).

[7] M. Fu, et al.: "Efficiency and optimal loads analysis for multiple-receiver wireless power transfer systems," IEEE Trans. Microw. Theory Techn. 63 (2015) 801 (DOI: 10.1109/TMTT.2015.2398422).

[8] S. Kim and B. Lee: "Analysis of efficiencies for multiple-input multiple-output 
wireless power transfer systems," J. Electromagn. Eng. Sci. 16 (2016) 126 (DOI: 10.5515/JKIEES.2016.16.2.126).

[9] T. Imura: "Cross-coupling canceling method for wireless power transfer via magnetic resonance coupling," IEEJ Trans. Industry Appl. 134 (2014) 564 (in Japanese) (DOI: 10.1541/ieejias.134.564).

[10] C. Zhong, et al.: "Reactance compensation method to eliminate cross coupling for two-receiver wireless power transfer system," IEICE Electron. Express 12 (2015) 20150016 (DOI: 10.1587/elex.12.20150016).

[11] D. Ahn and S. Hong: "Effect of coupling between multiple transmitters or multiple receivers on wireless power transfer," IEEE Trans. Ind. Electron. 60 (2013) 2602 (DOI: 10.1109/TIE.2012.2196902).

[12] S. Boyd and L. Vandenberghe: Convex Optimization (Cambridge University Press, 2004) 2nd ed. 140.

\section{Introduction}

Efficiency maximization based on load optimization for single-receiver inductive power transfer (IPT) has been studied extensively. Efficiency maximization for purely inductive coupling was investigated in [1]. [2, 3] then extended the solution for general IPT system, in which the coupling can be purely reactive (through air) or conductive (through tissue or biological media). Maximum efficiency is then mathematically formulated based on passive two-port network model in [4]. These studies yield insights into the system behaviors and, at the same time, provided powerful tools for analyzing more complicated IPT systems.

Efficiency maximization for multiple-receiver IPT is one of the most attractive topic. Solution for system with two receivers is studied in [5] and then extended in $[6,7,8]$ for system with arbitrary number of receivers. These works showed that the efficiency increases monotonically with the number of receivers, meaning that simultaneous charing is more efficient than charging in a time division manner. As $[6,7,8]$ optimized only resistances of the loads, these works, however, do not answer an important question that whether simultaneous resonance at the receiving coils gives the maximum efficiency. Cross-coupling among receivers is an important issue which has been investigated experimentally in literatures $[9,10,11]$. However, to the best of our knowledge, closed-form expression of optimal loads for a general scenario has not been derived so far. Furthermore, the formulations reported in literatures do not consider the case of coupling through conductive media. Resolving these issues requires the challenging joint optimization of both resistive and reactive components of the loads.

This paper aims to derive closed-form expressions of optimal loads and maximum efficiency for a general multiple-receiver IPT system. We model the system by passive $N$-port network and jointly optimize load resistances and reactances by applying the first-order necessary condition [12]. Verified by numerical analysis, our expression can be used to predict RF-to-RF efficiency for IPT system with any number of receivers, under any coupling condition and via any type of media. 


\section{System model and problem formulation}

\subsection{System model}

We consider a single frequency IPT system with one transmitter and $(N-1)$ receivers as illustrated in Fig. 1. The coils are surrounded by arbitrary media and there exists cross-coupling among the receiving coils. The system can be modeled by linear reciprocal $N$-port network where port 1 is the input and ports 2 to $N$ are the outputs. In practice, port 1 is usually connected to a sinusoidal power source and $(N-1)$ passive loads are connected to ports from 2 to $N$. The power transfer efficiency of the system, or RF-to-RF efficiency, is the ratio of total output power from ports 2 to $N$ to input power to port 1. The efficiency depends on the load impedances and the $\mathrm{N}$-port network parameters. In order to achieve the maximum efficiency, one need to be able to freely choose the values of load impedances. In practice, load impedances basically vary when the loads are in operations. Therefore, matching networks or DC-DC converters are usually inserted between the passive loads and the output ports in order to convert load impedances to the optimal values. Here, since we are interested in the values of loads which is seen from the output ports, we express the load impedance by voltage-to-current ratios and hide the power source, actual passive loads and matching networks from Fig. 1.

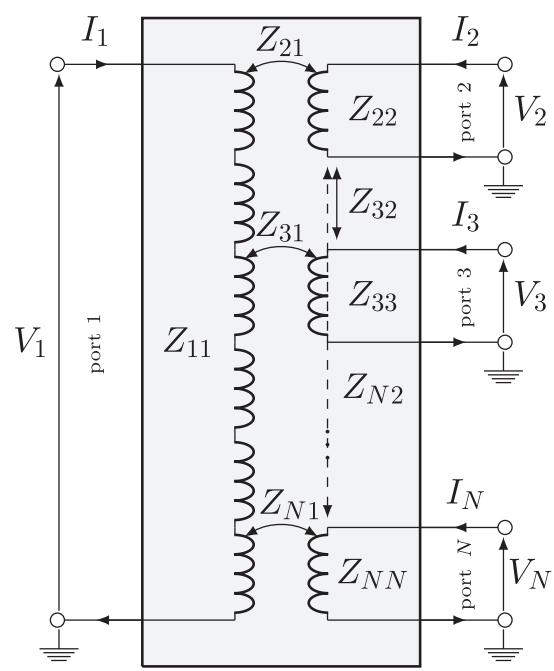

Fig. 1. System model of general multiple-receiver IPT.

Current-voltage relation of the system is expressed by Z-parameters as

$$
\left[\begin{array}{c}
V_{1} \\
V_{2} \\
\vdots \\
V_{N}
\end{array}\right]=\left[\begin{array}{cccc}
Z_{11} & Z_{12} & \ldots & Z_{1 N} \\
Z_{21} & Z_{22} & \ldots & Z_{2 N} \\
\vdots & \vdots & \ddots & \vdots \\
Z_{N 1} & Z_{N 2} & \ldots & Z_{N N}
\end{array}\right]\left[\begin{array}{c}
I_{1} \\
I_{2} \\
\vdots \\
I_{N}
\end{array}\right],
$$

where $I_{1}, V_{1}$ are complex-valued current and voltage phasors of the input port and $I_{n}, V_{n}(n \in\{2, \ldots, N\})$ are those of the outputs. In this model, we characterize the coupling between output port $n(n \in\{2, \ldots, N\})$ and input port 1 by the term $Z_{n 1}=R_{n 1}+j X_{n 1}$, where $R_{n 1}$ and $X_{n 1}$ respectively demonstrate the conductive 
(or direct) path and the inductive path of the coupling. The cross-coupling among receiving coils is represented by the term $Z_{n m}=R_{n m}+j X_{n m}$, where $m \in$ $\{2, \ldots, N\}$ and $m \neq n$. Load impedance at the output port $n$ is determined by $Z_{n}^{\mathrm{L}}=-V_{n} / I_{n}$.

\subsection{Problem formulation}

Without loss of generality, we take $I_{1}$ as the reference phasor and formulate the efficiency in terms of $I_{n}$. From Eq. (1), the real part of $V_{1}$ can be expressed as

$$
V_{1}^{\mathrm{re}}=R_{11} I_{1}+\sum_{n=2}^{N}\left(R_{n 1} I_{n}^{\mathrm{re}}-X_{n 1} I_{n}^{\mathrm{im}}\right),
$$

where $I_{n}^{\text {re }}$ and $I_{n}^{\mathrm{im}}$ are the real and imaginary parts of $I_{n}$, respectively. Real and imaginary parts of $V_{n}$ are

$$
\begin{gathered}
V_{n}^{\mathrm{re}}=R_{n 1} I_{1}+\sum_{m=2}^{N}\left(R_{n m} I_{m}^{\mathrm{re}}-X_{n m} I_{m}^{\mathrm{im}}\right), \\
V_{n}^{\mathrm{im}}=X_{n 1} I_{1}+\sum_{m=2}^{N}\left(R_{n m} I_{m}^{\mathrm{im}}+X_{n m} I_{m}^{\mathrm{re}}\right) .
\end{gathered}
$$

Therefore, input power to port 1 is given by

$$
P_{1}=\frac{1}{2} V_{1}^{\mathrm{re}} I_{1}=\frac{1}{2}\left[R_{11} I_{1}^{2}+\sum_{n=2}^{N} I_{1}\left(R_{n 1} I_{n}^{\mathrm{re}}-X_{n 1} I_{n}^{\mathrm{im}}\right)\right] .
$$

The output power from port $n$ is

$$
P_{n}=-\frac{1}{2}\left(V_{n}^{\mathrm{re}} I_{n}^{\mathrm{re}}+V_{n}^{\mathrm{im}} I_{n}^{\mathrm{im}}\right),
$$

thus the total output power is determined by

$$
\begin{aligned}
P_{\text {out }}=\sum_{n=2}^{N} P_{n}= & -\frac{1}{2} \sum_{n=2}^{N}\left(R_{n 1} I_{1} I_{n}^{\mathrm{re}}+X_{n 1} I_{1} I_{n}^{\mathrm{im}}\right) \\
& -\frac{1}{2} \sum_{n=2}^{N} \sum_{m=2}^{N} R_{n m}\left(I_{n}^{\mathrm{re}} I_{m}^{\mathrm{re}}+I_{n}^{\mathrm{im}} I_{m}^{\mathrm{im}}\right) .
\end{aligned}
$$

Finally, RF-to-RF efficiency is formulated as

$$
\eta=\frac{P_{\mathrm{out}}}{P_{1}}=\frac{\sum_{n=2}^{N}\left(R_{n 1} I_{1} I_{n}^{\mathrm{re}}+X_{n 1} I_{1} I_{n}^{\mathrm{im}}\right)+\sum_{n=2}^{N} \sum_{m=2}^{N} R_{n m}\left(I_{n}^{\mathrm{re}} I_{m}^{\mathrm{re}}+I_{n}^{\mathrm{im}} I_{m}^{\mathrm{im}}\right)}{R_{11} I_{1}^{2}+\sum_{n=2}^{N} I_{1}\left(R_{n 1} I_{n}^{\mathrm{re}}-X_{n 1} I_{n}^{\mathrm{im}}\right)} .
$$

In Eq. (8), $I_{1}$ is a parameter. The efficiency $\eta$ can be viewed as a function of $I_{n}^{\mathrm{re}}$ and $I_{n}^{\mathrm{im}}$. The output currents $I_{n}^{\mathrm{re}}$ and $I_{n}^{\mathrm{im}}$ take values from $-\infty$ to $\infty$ given that all output power is non-negative, i.e., $P_{n} \geq 0 \forall n \in\{2, \ldots, N\}$.

\section{Maximum efficiency and optimal loads}

We derive the maximum value of the multi-variable function $\eta$ by applying the firstorder necessary condition [12] on $I_{n}^{\mathrm{re}}$ and $I_{n}^{\mathrm{im}}$. When the condition is satisfied, $\forall n \in\{2, \ldots, N\}$, we have 


$$
\frac{\partial \eta}{\partial I_{n}^{\mathrm{re}}}=0, \quad \frac{\partial \eta}{\partial I_{n}^{\mathrm{im}}}=0 .
$$

From equations (8) and (9) it follows a system of $2 n$ simultaneous equations. Solving this system of equations gives us the current values, from which we can derive the maximum efficiency. Since it is very complicated to solve these equations directly, we do this in a subtle way as follows.

$$
\begin{aligned}
\frac{\partial \eta}{\partial I_{n}^{\mathrm{re}}} & =\frac{\left(\partial P_{\mathrm{out}} / \partial I_{n}^{\mathrm{re}}\right) P_{1}-\left(\partial P_{1} / \partial I_{n}^{\mathrm{re}}\right) P_{\mathrm{out}}}{P_{1}^{2}}, \\
\frac{\partial \eta}{\partial I_{n}^{\mathrm{im}}} & =\frac{\left(\partial P_{\mathrm{out}} / \partial I_{n}^{\mathrm{im}}\right) P_{1}-\left(\partial P_{1} / \partial I_{n}^{\mathrm{im}}\right) P_{\mathrm{out}}}{P_{1}^{2}} .
\end{aligned}
$$

From this it follows that $\forall n \in\{2, \ldots, N\}$,

$$
\begin{aligned}
& \sum_{m=2}^{N} R_{n m} I_{m}^{\mathrm{re}}=-R_{n 1} \frac{(1+\eta) I_{1}}{2} \\
& \sum_{m=2}^{N} R_{n m} I_{m}^{\mathrm{im}}=-X_{n 1} \frac{(1-\eta) I_{1}}{2}
\end{aligned}
$$

Let

$$
\begin{aligned}
\boldsymbol{R}_{\mathrm{rr}} & =\left[\begin{array}{ccc}
R_{22} & \ldots & R_{2 N} \\
\vdots & \ddots & \vdots \\
R_{N 2} & \ldots & R_{N N}
\end{array}\right], \\
\boldsymbol{R}_{\mathrm{rt}} & =\left[\begin{array}{c}
R_{21} \\
\vdots \\
R_{N 1}
\end{array}\right], \quad \boldsymbol{X}_{\mathrm{rt}}=\left[\begin{array}{c}
X_{21} \\
\vdots \\
X_{N 1}
\end{array}\right], \quad \boldsymbol{I}_{\mathrm{r}}^{\mathrm{re}}=\left[\begin{array}{c}
I_{2}^{\mathrm{re}} \\
\vdots \\
I_{N}^{\mathrm{re}}
\end{array}\right], \quad \boldsymbol{I}_{\mathrm{r}}^{\mathrm{im}}=\left[\begin{array}{c}
I_{2}^{\mathrm{im}} \\
\vdots \\
I_{N}^{\mathrm{im}}
\end{array}\right] .
\end{aligned}
$$

Equations (12) and (13) and be expressed as

$$
\begin{aligned}
& \boldsymbol{I}_{\mathrm{r}}^{\mathrm{re}}=-\boldsymbol{R}_{\mathrm{rr}}^{-1} \boldsymbol{R}_{\mathrm{rt}}(1+\eta) I_{1} / 2, \\
& \boldsymbol{I}_{\mathrm{r}}^{\mathrm{im}}=-\boldsymbol{R}_{\mathrm{rr}}^{-1} \boldsymbol{X}_{\mathrm{rt}}(1-\eta) I_{1} / 2 .
\end{aligned}
$$

Substituting Eq. (15) and Eq. (16) in to Eq. (8), we get an equation of $\eta$.

$$
\frac{A+B}{4} \eta^{2}-\left(1-\frac{A-B}{2}\right) \eta+\frac{A+B}{4}=0
$$

which yields two solutions

$$
\eta=\frac{(\sqrt{1+A}-\sqrt{1-B})(\sqrt{1+A}+\sqrt{1-B})}{(\sqrt{1+A} \pm \sqrt{1-B})^{2}},
$$

where

$$
A=\frac{1}{R_{11}} \boldsymbol{X}_{\mathrm{rt}}^{\mathrm{T}} \boldsymbol{R}_{\mathrm{rr}}^{-1} \boldsymbol{X}_{\mathrm{rt}}, \quad B=\frac{1}{R_{11}} \boldsymbol{R}_{\mathrm{rt}}^{\mathrm{T}} \boldsymbol{R}_{\mathrm{rr}}^{-1} \boldsymbol{R}_{\mathrm{rt}} .
$$

The condition that $0 \leq \eta<1$ is satisfied only with the solution received when replacing the " \pm " symbol in Eq. (18) by " $-"$. This solution is the maximum efficiency 


$$
\eta_{\max }=\frac{\sqrt{1+A}-\sqrt{1-B}}{\sqrt{1+A}+\sqrt{1-B}} .
$$

When the efficiency reaches its maximum, port currents are given by

$$
\left[\begin{array}{c}
I_{2}^{*} \\
\vdots \\
I_{N}^{*}
\end{array}\right]=-\boldsymbol{R}_{\mathrm{rr}}^{-1}\left[\boldsymbol{R}_{\mathrm{rt}}\left(1+\eta_{\max }\right)+j \boldsymbol{X}_{\mathrm{rt}}\left(1-\eta_{\max }\right)\right] I_{1} / 2 .
$$

And port voltages $V_{n}^{*}=Z_{n 1} I_{1}+\sum_{m=2}^{N} Z_{n m} I_{m}^{*}$. Thus, the optimal loads are

$$
Z_{n}^{\mathrm{L}(\mathrm{opt})}=R_{n}^{\mathrm{L}(\mathrm{opt})}+j X_{n}^{\mathrm{L}(\mathrm{opt})}=-V_{n}^{*} / I_{n}^{*}=-\frac{Z_{n 1} I_{1}+\sum_{m=2}^{N} Z_{n m} I_{m}^{*}}{I_{n}^{*}} .
$$

\section{Case study}

\subsection{IPT through air and without cross-coupling}

Coupling through air is characterized by $\boldsymbol{R}_{\mathrm{rt}}=\mathbf{0}, \boldsymbol{R}_{\mathrm{rr}}=\operatorname{diag}\left(R_{22}, \ldots, R_{N N}\right)$ and the condition of no cross-coupling is characterized by $X_{n m}=0(\forall m \neq n)$. In this case, $A=\frac{1}{R_{11}} \sum_{n=2}^{N} \frac{X_{n 1}^{2}}{R_{n n}}, B=0$ and the maximum efficiency becomes

$$
\eta_{\max }=\frac{\sqrt{1+\frac{1}{R_{11}} \sum_{n=2}^{N} \frac{X_{n 1}^{2}}{R_{n n}}}-1}{\sqrt{1+\frac{1}{R_{11}} \sum_{n=2}^{N} \frac{X_{n 1}^{2}}{R_{n n}}}+1} .
$$

The resistance and reactance of the optimal load are

$$
\begin{aligned}
& R_{n}^{\mathrm{L}(\mathrm{opt})}=R_{n n} \sqrt{1+\frac{1}{R_{11}} \sum_{n=2}^{N} \frac{X_{n 1}^{2}}{R_{n n}},} \\
& X_{n}^{\mathrm{L}(\mathrm{opt})}=-X_{n n} .
\end{aligned}
$$

Maximum efficiency and optimal load resistance given by equations (23) and (24) matches perfectly with the formulations in $[6,7,8]$. Optimal load reactance determined by Eq. (25) confirms the optimality of the common practice of resonating out the receiving coils. As will be shown in sections 4.2 and 4.3, this common practice loses its optimality when coupling is through conductive media and/or when the cross-coupling exists.

\subsection{IPT through air and with cross-coupling}

In this case, also $\boldsymbol{R}_{\mathrm{rt}}=\mathbf{0}, \boldsymbol{R}_{\mathrm{rr}}=\operatorname{diag}\left(R_{22}, \ldots, R_{N N}\right)$, thus $A=\frac{1}{R_{11}} \sum_{n=2}^{N} \frac{X_{n 1}^{2}}{R_{n n}}$ and $B=0$. As a result,

$$
\eta_{\max }=\frac{\sqrt{1+\frac{1}{R_{11}} \sum_{n=2}^{N} \frac{X_{n 1}^{2}}{R_{n n}}}-1}{\sqrt{1+\frac{1}{R_{11}} \sum_{n=2}^{N} \frac{X_{n 1}^{2}}{R_{n n}}}+1}
$$




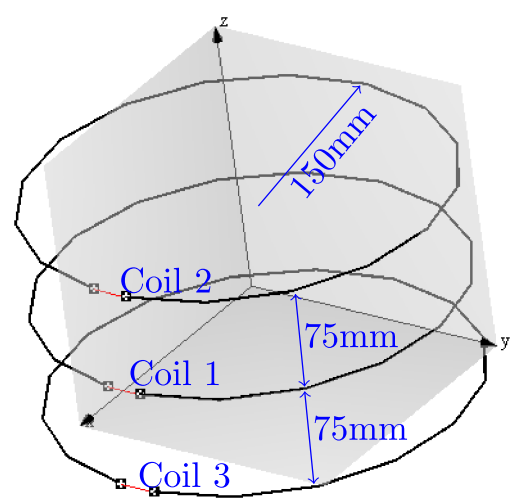

Fig. 2. Two-receiver IPT system with cross-coupling.

$$
R_{n}^{\mathrm{L}(\mathrm{opt})}=R_{n n} \sqrt{1+\frac{1}{R_{11}} \sum_{n=2}^{N} \frac{X_{n 1}^{2}}{R_{n n}}}
$$

This result implies that the cross-coupling does not affect the maximum efficiency and optimal load resistance. However, in this case, since $X_{n m} \neq 0$ $(\forall m \neq n)$, the optimal load reactance becomes

$$
X_{n}^{\mathrm{L}(\mathrm{opt})}=-X_{n n}-\sum_{m=2, m \neq n}^{N} X_{n m} \frac{X_{m 1} R_{n n}}{X_{n 1} R_{m m}},
$$

meaning that resonating out the receiving coils is not optimal under the existence of cross-coupling. Another important observation can be drawn from Eq. (26) is that the efficiency also increases with the number of receivers.

In order to verify the effectiveness of equations (26)-(28), we perform electromagnetic field analysis for an IPT system with two receivers shown in Fig. 2. In this analysis, all coils are made from copper wire with diameter of $1 \mathrm{~mm}$ and with conductivity of $5.8 \times 10^{7} \mathrm{~S} / \mathrm{m}$. Operating frequency $f=13.56 \mathrm{MHz}$, which is an industrial scientific and medical (ISM) band. In this system, coil 1 is the transmitting coil while coil 2 and coil 3 are the receiving. Sizes and positions of coils are illustrated in the figure. By computer simulation, Z-parameters for the system are observed as

$$
\boldsymbol{Z}=\left[\begin{array}{ccc}
0.39+j 94.22 & j 14.03 & j 14.03 \\
j 14.03 & 0.39+j 94.21 & j 6.21 \\
j 14.03 & j 6.21 & 0.39+j 94.21
\end{array}\right]
$$

indicating that there exists cross-coupling between coils 2 and 3 as $Z_{23}=$ $Z_{32}=j 6.21[\Omega]$. We then carry out an exhaustive search to find the optimal loads as well as maximum efficiency. Because receiving coils have the same size and distance to the transmitting coil, we assume all loads as $Z_{2}^{\mathrm{L}}=Z_{3}^{\mathrm{L}}=R^{\mathrm{L}}+j X^{\mathrm{L}}$. We varied the loads $R^{\mathrm{L}}, X^{\mathrm{L}}$ and obtained efficiency $\eta$ as shown in Fig. 3. The simulation result shows that optimal loads and maximum efficiency are $20-j 100$ $[\Omega]$ and $\eta=0.96$. These values are very tight to the theoretically optimal values $19.85-j 100[\Omega]$ and $\eta_{\max }=0.96$ calculated by equations (26)-(28) with Zparameters given in Eq. (29). 


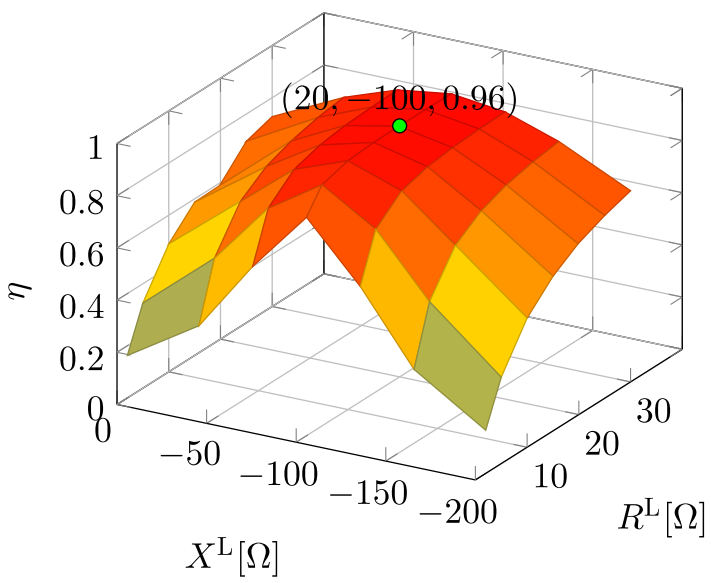

Fig. 3. Efficiency v.s. load resistance and reactance.

\subsection{IPT through conductive media and without cross-coupling}

Coupling through conductive media is characterized by $\boldsymbol{R}_{\mathrm{rt}} \neq \mathbf{0}$. Thus $A=\frac{1}{R_{11}} \sum_{n=2}^{N} \frac{X_{n 1}^{2}}{R_{n n}}, B=\frac{1}{R_{11}} \sum_{n=2}^{N} \frac{R_{n 1}^{2}}{R_{n n}}$ and

$$
\begin{aligned}
\eta_{\max } & =\frac{\sqrt{1+\frac{1}{R_{11}} \sum_{n=2}^{N} \frac{X_{n 1}^{2}}{R_{n n}}}-\sqrt{1-\frac{1}{R_{11}} \sum_{n=2}^{N} \frac{R_{n 1}^{2}}{R_{n n}}}}{\sqrt{1+\frac{1}{R_{11}} \sum_{n=2}^{N} \frac{X_{n 1}^{2}}{R_{n n}}}+\sqrt{1-\frac{1}{R_{11}} \sum_{n=2}^{N} \frac{R_{n 1}^{2}}{R_{n n}}}} \\
R_{n}^{\mathrm{L}(\mathrm{opt})} & =\frac{\left(1-\eta_{\max }^{2}\right)\left(R_{n 1}^{2}+X_{n 1}^{2}\right) R_{n n}}{\left(1+\eta_{\max }\right)^{2} R_{n 1}^{2}+\left(1-\eta_{\max }\right)^{2} X_{n 1}^{2}}, \\
X_{n}^{\mathrm{L}(\mathrm{opt})} & =\frac{4 \eta_{\max } R_{n 1} X_{n 1} R_{n n}}{\left(1+\eta_{\max }\right)^{2} R_{n 1}^{2}+\left(1-\eta_{\max }\right)^{2} X_{n 1}^{2}}-X_{n n} .
\end{aligned}
$$

One should note that $\eta_{\max }$ in equations (31) and (32) is determined by Eq. (30). This result indicates that even when the coupling is via conductive media, the efficiency also increases with the number of receivers. However, resonating out the output ports loses its optimality as Eq. (32) shows that the optimal reactance is different from $-X_{n n}$.

Numerical analysis is performed for an IPT system with two receivers shown in Fig. 4. In this system, all coils are made from $1 \mathrm{~mm} \times 1 \mathrm{~mm}$ square solid rods of copper with conductivity of $5.8 \times 10^{7} \mathrm{~S} / \mathrm{m}$ and the operating frequency $f=$ 13.56 MHz. Coil 1 is the transmitting coil while coil 2 and coil 3 are the receiving. All coils are placed on $2 \mathrm{~mm}$-thick acrylic substrates with relative permittivity of 2.6 and conductivity of $1.4 \times 10^{-5} \mathrm{~S} / \mathrm{m}$. The medium between transmitting and receiving coils is sea water with relative permittivity of 81.0 and conductivity of $5 \mathrm{~S} / \mathrm{m}$. In this investigation, thickness of the medium is varied as $d=10,15,20$, 25, $30 \mathrm{~mm}$.

Z-parameters for the three-port network are obtained via simulation and shown in Table I. The norm of trans-impedance between ports 2 and 3 is observed to be very small, confirming that the cross-coupling between is negligible. Because receiving coils have the same size, we assume loads impedances as $Z_{2}^{\mathrm{L}}=Z_{3}^{\mathrm{L}}=$ $R^{\mathrm{L}}+j X^{\mathrm{L}}$. Fig. 5 shows the variation of $\eta$ with $R^{\mathrm{L}}$ and $X^{\mathrm{L}}$ when $d=10 \mathrm{~mm}$. The simulation result shows that optimal loads and maximum efficiency are $Z^{\mathrm{L}}=$ 


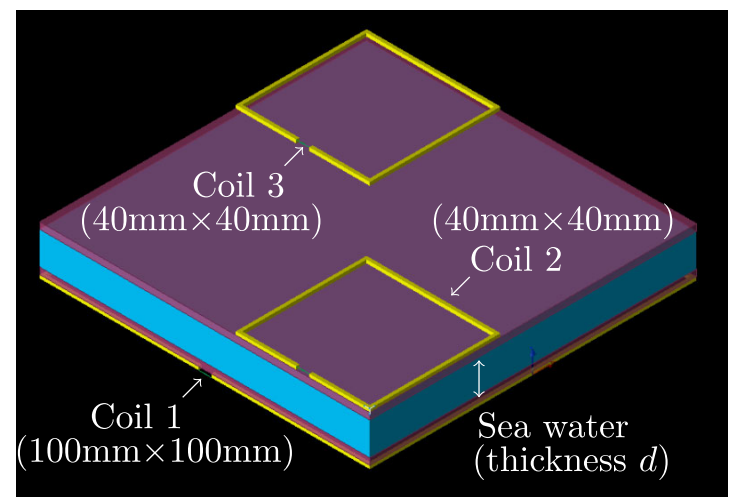

Fig. 4. Two-receiver IPT through conductive media without crosscoupling.

Table I. Z-parameters measured by simulation of system in Fig. 4.

\begin{tabular}{c|ccccc}
\hline$d[\mathrm{~mm}]$ & 10 & 15 & 20 & 25 & 30 \\
\hline$R_{11}[\Omega]$ & 0.30 & 0.38 & 0.44 & 0.48 & 0.51 \\
\hline$X_{11}[\Omega]$ & 26.79 & 26.79 & 26.80 & 26.80 & 26.79 \\
\hline$R_{22}[\Omega]$ & 0.06 & 0.06 & 0.07 & 0.07 & 0.07 \\
\hline$X_{22}[\Omega]$ & 8.25 & 8.24 & 8.25 & 8.25 & 8.25 \\
\hline$R_{33}[\Omega]$ & 0.06 & 0.06 & 0.07 & 0.07 & 0.07 \\
\hline$X_{33}[\Omega]$ & 8.24 & 8.25 & 8.25 & 8.25 & 8.25 \\
\hline$R_{21}[\Omega]$ & 0.03 & 0.04 & 0.04 & 0.04 & 0.04 \\
\hline$X_{21}[\Omega]$ & 1.32 & 1.04 & 0.85 & 0.71 & 0.60 \\
\hline$R_{31}[\Omega]$ & 0.03 & 0.04 & 0.04 & 0.04 & 0.04 \\
\hline$X_{31}[\Omega]$ & 1.33 & 1.05 & 0.86 & 0.71 & 0.60 \\
\hline
\end{tabular}

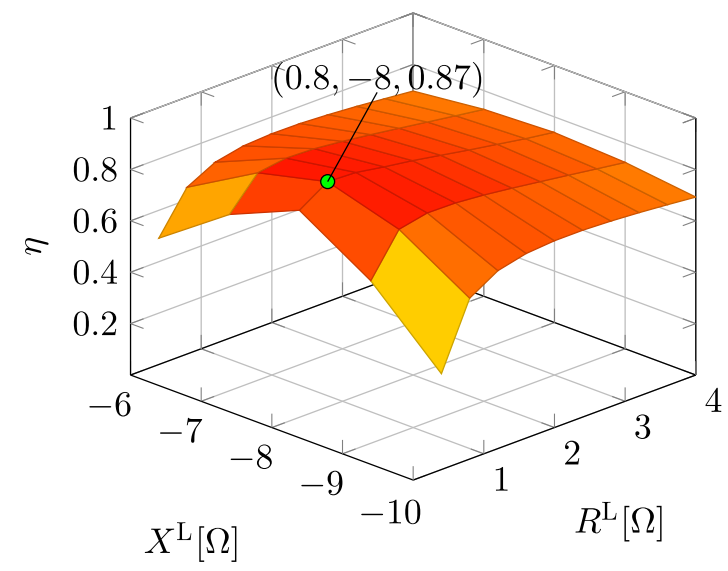

Fig. 5. Efficiency v.s. load resistance and reactance when $d=10 \mathrm{~mm}$.

$0.80-j 8.0[\Omega]$ and $\eta=0.87$, respectively. These values are very close to the theoretically optimal value $Z^{\mathrm{L}(\mathrm{opt})}=0.77-j 7.99[\Omega], \eta_{\max }=0.88$ calculated by equations (30)-(32).

Fig. 6 demonstrates the simulated efficiency and the efficiency that is theoretically estimated by Eq. (30) for various values of $d$. The two values are shown to be very tight to each other, confirming the optimality of the derived solutions. 


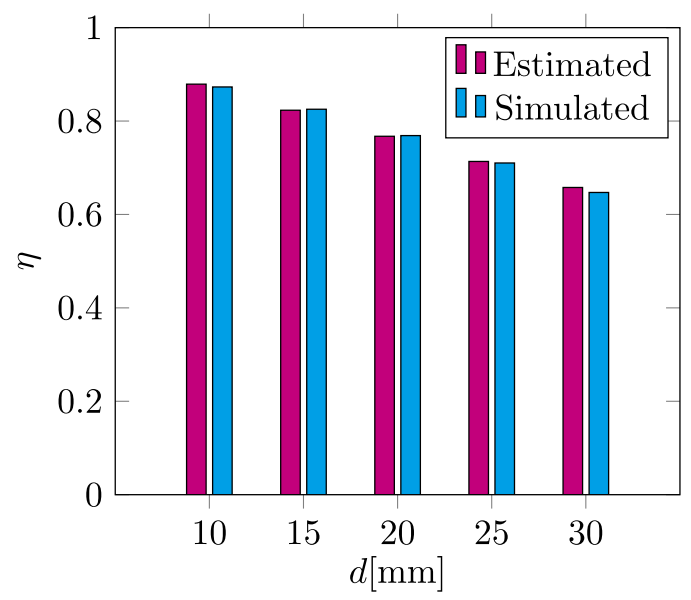

Fig. 6. Efficiency when load are optimized using equations (31) and (32).

\section{Conclusion and discussion}

This paper derived closed-form expressions of optimal loads that maximize RF-toRF efficiency for general multiple-receiver IPT system. Verified by numerical analysis, the derived expressions give some interesting insights into the system behavior as follows.

- Cross-coupling, in fact, does not affect the maximum efficiency. Many previous investigations have reported that cross-coupling decreases the system efficiency. However, in such investigations the loads are not optimal. As mathematically shown in section 4.2 , when the loads are optimized by the derived expressions, the impact of cross-coupling can be perfectly mitigated.

- Resonating out the receiving coils is optimal only for the case of coupling through air and without cross-coupling among receivers. This common practice loses its optimality when the media is conductive and/or when the crosscoupling exists.

- The maximum efficiency increases with the number of receivers, regardless of coupling condition and/or electrical property of the surrounding media.

Experimental studies are required to further confirm the derived expression as well as the aforementioned observations.

\section{Acknowledgments}

This work was supported by DAIHEN Corporation. 